\title{
Analysis of Power Consumption on Communication Methods for the Emergency Deployment System
}

\author{
JaeMin Hong, WenJun Zhu, KyuJin Kim, Shinheon Kim, and ChongGun Kim
}

\begin{abstract}
Energy consumption phenomena of IOT based emergency deployment system which used in general hospitals and other institutions are analyzed. The system can treat realtime emergency messages between servers and mobile clients by using multiple communication methods. Especially in the hospital environment, in order to avoid patient anxiety caused by emergency situations, the system informs sends the emergency situation to the person who is to response the situation by using one-way broadcast communications. After that a two-way communications and indoor positioning methods are used to control and record the response processes.
\end{abstract}

Keywords-Bluetooth LE, Wi-Fi, Power consumption

\section{Introduction}

In a general hospital environment, in case of emergency, it is needed to send the emergency information in real time to the persons in charge by using SMS or telephone call for respond the situation. The persons in charge with the corresponding situation have to respond as soon as possible. Based on the general emergency call method, if the emergency information cannot be timely conveyed to the persons in charge, there will delayed the deployment of the persons in charge and cannot respond be in time. A system that can send emergency information to persons in charge by using multiple communication methods when an emergency situation occurs and the persons in charge can respond to the emergency in real time is proposed. The system is also can record and manage all emergency activity information.

Figure 1 shows the multiple communication concept of the proposed system.

JaeMin Hong. Dept. of Computer Engineering, Yeungnam Univ.

WenJun Zhu. Dept. of Computer Engineering, Yeungnam Univ.

KyuJin Kim. Dept. of Nursing, Kyungpook National University.

ShinHeon Kim. Dept. of Computer Engineering, Yeungnam Univ.

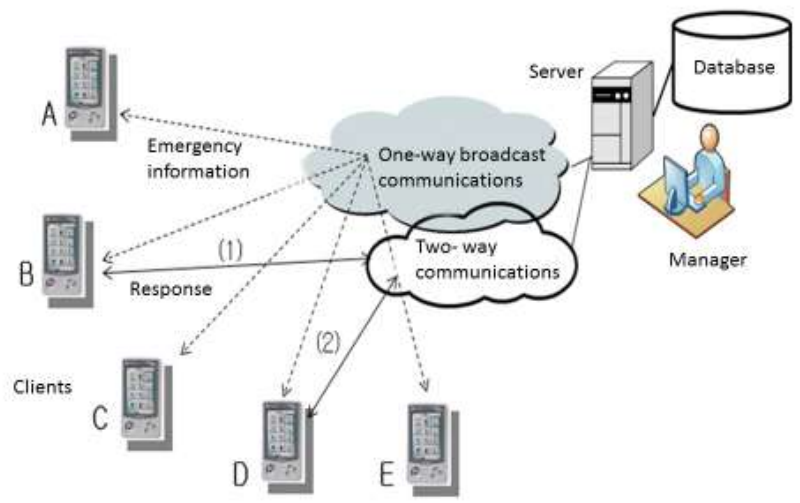

Figure 1. The communication concept of the proposed system

\section{Previous and related studies}

In this study, the management server uses one-way broadcast communication to send emergency alarm message to persons in charge, according to the emergency reaction protocol, then change to two-way communications by confirming from the client to exchange emergency information with clients, all the activities and position of the responder are tracked in real time.

Bluetooth LE (Bluetooth Low Energy), visible light communication technology, SMS message can be used as one-way broadcast communication method, Wi-Fi and cellular mobile communications can be used as two-way TCP communication methods in this system.

\section{A. Bluetooth $\mathbf{L E}$}

By growing importance of IoT (Internet of Things), Bluetooth LE application is also diffused. Compared with the Bluetooth, it does not need to maintain a long connection, therefore power consumption is also greatly reduced.

BLE does not need pairing that can send data to multi clients in one-way, this process is called Advertising. All the terminals which Bluetooth enabled can receive the advertising data. When a message is transmitted via advertising, the message can be receiving by multi users in the same time.

The BLE device for advertising is called beacon and it is possible to indoor positioning by using RSSI (Received Signal Strength Indication) value of beacon signal [1]. 


\section{B. Two-way TCP socket communication based on Wi-Fi}

Socket communications have two major transport layer protocols, TCP (Transfer Control Protocol) and UDP (User Datagram Protocol).

The UDP communication has no handshaking dialogues among the processes for session, and cannot provide any reliability control so there is no guarantee of delivery, ordering, or duplicate protection of datagrams. The TCP communication provides reliable, ordered and error-checked delivery of a stream of octets between processes which are running on hosts based on IP network. In this study, TCP is used for socket communications.

A TCP communication is requested by using the IP addresses and port numbers of two processes. The client starts the connection and if the server does not accept the response, the connection will be failed. If connection is created, data can be exchanged through the socket between the server and client. The TCP can provide error control and flow control, if the data not received properly, it can be requested again [2].

\section{Indoor positioning by using Wi-Fi signal strength}

GPS (Global Positioning System) is the most widely used outdoor positioning system. But in the indoor environment, GPS cannot work properly. Therefore, many research of indoor positioning are in progress. For example, visible light communications [3], BLE signal strength [1][9], Wi-Fi signal strength and so on can be used [4][5] For finding the coordinate of mobile object in indoor environment, fingerprint and triangulation methods are widely used.

The triangulation method is a geometric method of computing a coordinate by calculating each distance from three reference points. It is necessary to be able to obtain the correct distance from the wireless AP as reference point so that the correct position can be traced [10].

Fingerprint method is RSSI (Received Signal Strength Indication)-based, but it simply relies on the previously recorded data of the signal strength from several reference access points in the proper range. Storing this information in a database along with the known coordinates of the tracking device is clone in an offline phase. During the online position decision phase, the current RSSI vector at an unknown location is compared to those stored in the fingerprint $\mathrm{DB}$ and the closest matching position is returned as the estimated user location [8].

Wi-Fi or BLE signal strength can be used for calculating distance for using the fingerprint method.

\section{Emergency Deployment System in a general hospital}

When a patient meet in an emergency situation, this system can send the emergency notice to the persons in charge on various departments, then by the confirming to the notice which means the corresponding departments can give an initial solution. Indoor positioning is used to guide the persons in charge to the current location of the patient, then lead those on the most appropriate route to the operating room.

\section{Comparison of power consumption on Wi-Fi and BLE}

Different communication methods not only have difference on data rate and communication distance, power consumptions are also different. Therefore, the power consumption tendencies are seriously. Considered in the proposed system.

\section{A. Power consumptions based on hardware}

The WL18xxMOD WiLink 8 Single band Combo Module developed by TI which is widely used in portable mobile devices has be selected for power consumption analysis of Wi-Fi device. One important selection reason is the chip on the device uses the same antenna for Wi-Fi and Bluetooth communications.

The chip's operating power of Wi-Fi communication showed on Table 1[6]. It can be seen that, depending on the negotiation data rate and the encoding method, the operating power of chip at $49 \mathrm{~mA}$ to $85 \mathrm{~mA}$ when the chip used as a Wi-Fi receiver. As a Wi-Fi transmitter, the operating power of chip surged to $238 \mathrm{~mA}$ to $420 \mathrm{~mA}$.

Table 1. Operating current of Wi-Fi communication

\begin{tabular}{|c|c|c|c|}
\hline & SPECIFICATION ITEMS & \begin{tabular}{|c|} 
TYP $(\mathrm{AVG})$ \\
$-25^{\circ} \mathrm{C}$
\end{tabular} & UNITS \\
\hline \multirow{11}{*}{ Receiver } & $\begin{array}{l}\text { Low-power mode (LPM) } \\
\text { 2.4GHz RX SISO20 single } \\
\text { chain }\end{array}$ & 49 & \multirow{17}{*}{$\mathrm{mA}$} \\
\hline & 2.4 GHz RX search SISO20 & 54 & \\
\hline & $2.4 \mathrm{GHz}$ RX search MIMO20 & 74 & \\
\hline & 2.4 GHz RX search SISO40 & 59 & \\
\hline & $\begin{array}{l}2.4 \mathrm{GHz} \text { RX } 20 \text { M SISO } 11 \\
\text { CCK }\end{array}$ & 56 & \\
\hline & $\begin{array}{c}2.4 \mathrm{GHz} \text { RX } 20 \text { M SISO } 6 \\
\text { OFDM }\end{array}$ & 61 & \\
\hline & $\begin{array}{c}2.4 \mathrm{GHz} \text { RX } 20 \text { M SISO } \\
\text { MCS7 }\end{array}$ & 65 & \\
\hline & $\begin{array}{l}2.4 \mathrm{GHz} \text { RX } 20 \mathrm{M} \text { MRC } 1 \\
\text { DSSS }\end{array}$ & 74 & \\
\hline & $\begin{array}{c}2.4 \mathrm{GHz} \text { RX } 20 \text { M MRC } 6 \\
\text { OFDM }\end{array}$ & 81 & \\
\hline & $\begin{array}{c}2.4 \mathrm{GHz} \text { RX } 20 \mathrm{M} \text { MRC } 54 \\
\text { OFDM }\end{array}$ & 85 & \\
\hline & 2.4 GHz RX 40 M MCS7 & 77 & \\
\hline \multirow{6}{*}{ Transmitter } & $\begin{array}{c}2.4 \mathrm{GHz} \text { TX } 20 \mathrm{M} \mathrm{SISO} \\
\text { 6OFDM } 15.4 \mathrm{dBm}\end{array}$ & 285 & \\
\hline & $\begin{array}{c}2.4 \mathrm{GHz} \text { TX } 20 \mathrm{M} \text { SISO } 11 \\
\text { CCK } 15.4 \mathrm{dBm}\end{array}$ & 273 & \\
\hline & \begin{tabular}{|c|}
$2.4 \mathrm{GHz}$ TX $20 \mathrm{M}$ SISO 54 \\
ODFM $12.7 \mathrm{dBm}$ \\
\end{tabular} & 247 & \\
\hline & $\begin{array}{c}\text { 2.4GHz TX } 20 \text { M SISO MCS7 } \\
11.2 \mathrm{dBm} \\
\end{array}$ & 238 & \\
\hline & \begin{tabular}{|c|} 
2.4GHz TX $20 \mathrm{M}$ MIMO \\
MCS15 $11.2 \mathrm{dBm}$ \\
\end{tabular} & 420 & \\
\hline & $\begin{array}{c}2.4 \mathrm{GHz} \text { TX } 40 \mathrm{M} \text { SISO MCS7 } \\
8.2 \mathrm{dBm}\end{array}$ & 243 & \\
\hline
\end{tabular}


The chip's operating powers of Bluetooth BR (Basic Rate) and EDR (Enhanced Data Rate) communication showed on Table 2[6], The operating power of each case in a range from $178 \mu \mathrm{A}$ to $33 \mathrm{~mA}$.

Table 2. Operating currents of Bluetooth

\begin{tabular}{|c|c|c|}
\hline USE CASE & TYP & UNIT \\
\hline BR voice HV3 + sniff & 11.6 & $\mathrm{~mA}$ \\
\hline EDR voice $2-E V 3$ no retransmission + sniff & 5.9 & $\mathrm{~mA}$ \\
\hline Sniff 1 attempt $1.28 \mathrm{~s}$ & 178.0 & $\mu \mathrm{A}$ \\
\hline $\begin{array}{l}\text { EDR A2DP EDR2(master). SBC high quality } \\
\qquad-345 \mathrm{kbps}\end{array}$ & 10.4 & $\mathrm{~mA}$ \\
\hline $\begin{array}{l}\text { EDR A2DP EDR2(master). MP3 high quality } \\
\qquad-192 \mathrm{kbps}\end{array}$ & 7.5 & $\mathrm{~mA}$ \\
\hline Full throughput ACL RX: RX-2DH5 & 18.0 & $\mathrm{~mA}$ \\
\hline Full throughput BR ACL TX: TX-DH5 & 50.0 & $\mathrm{~mA}$ \\
\hline Full throughput EDR ACL TX: TX-2DH5 & 33.0 & $\mathrm{~mA}$ \\
\hline $\begin{array}{c}\text { Page scan or inquiry scan (scan interval is } \\
1.28 \mathrm{~s} \text { or } 11.25 \mathrm{~ms} \text {, respectively) }\end{array}$ & 253.0 & $\mu \mathrm{A}$ \\
\hline $\begin{array}{c}\text { Page scan and inquiry scan (scan interval is } \\
1.28 \mathrm{~s} \text { and } 2.56 \mathrm{~s} \text {, respectively) }\end{array}$ & 332.0 & $\mu \mathrm{A}$ \\
\hline
\end{tabular}

* Current is measured at output power as follows: BR at 11.7 $\mathrm{dBm} ; \mathrm{EDR}$ at $7.2 \mathrm{dBm}$

Table 3 showed the chip's operating powers of Bluetooth $\mathrm{LE}$ (BLE). The current values are from $124 \mu \mathrm{A}$ to $266 \mu \mathrm{A}$ [6].

The power consumption of Wi-Fi is a thousand times than that of Bluetooth LE.

Table 3. Operating current of Bluetooth LE(BLE)

\begin{tabular}{|c|c|c|}
\hline USE CASE & TYP & UNIT \\
\hline Advertising, not connectable & 131 & \\
\cline { 1 - 2 } Advertising, discoverable & 143 & \multirow{2}{*}{ NA } \\
\cline { 1 - 2 } Scanning & 266 & \\
\cline { 1 - 2 } Connected, master role, 1.28s connect interval & 124 & \\
\cline { 1 - 2 } Connected, slave role, 1.28s connect interval & 132 & \\
\cline { 1 - 2 } *All current measured at output power of $7.0 \mathrm{dBm}$ \\
\hline
\end{tabular}

\section{B. Power consumptions based on software}

The power consumption of TCP/IP based two-way communication protocol via $\mathrm{Wi}-\mathrm{Fi}$, one-way Bluetooth LE communications, and using Google Cloud Message (GCM) Push [7] are tested. The confirmation of power consumption software used in this study is done based on the BroadcastReceiver function of the Android operating system. Figure 2 is the flow chart of this program. When an event is transmitted from the Android system, the receiver application which is corresponded can be executed.

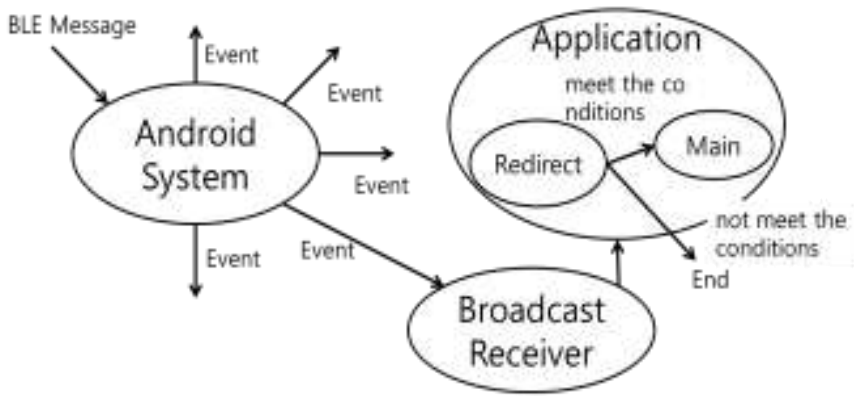

Figure 2. Flow chart of power consumption analysis program

In power consumption evaluation, data package sends total 60 times in 10 minutes. Then the power consumption results are measured after completion of communications. Samsung Galaxy S3 is used as receiver, ipTime A3004 wireless router is used as Wi-Fi transmitter, Raspberry Pi 3 is used as BLE transmitter in this experiment. The results of power consumption experiment are shown on Table 4

Table 4. Result of Power consumption experiment

\begin{tabular}{|c|c|c|c|}
\hline $\begin{array}{c}\text { Communication } \\
\text { method }\end{array}$ & $\begin{array}{c}\text { TCP/IP based own } \\
\text { Communication } \\
\text { protocol via Wi-Fi }\end{array}$ & Bluetooth LE & $\begin{array}{c}\text { GCM } \\
\text { Push }\end{array}$ \\
\hline Battery consumption & $9 \%$ & $2 \%$ & $1 \%$ \\
\hline
\end{tabular}

As shown at the tables, Bluetooth LE communication and GCM Push consumes much less power than Wi-Fi communication in both tests.

\section{Conclusions}

Various communication results for checking power consumption are evaluated. Considering hardware point of view, the chip's operating power is from $49 \mathrm{~mA}$ to $420 \mathrm{~mA}$ when Wi-Fi communication is in progress. The consumption power value of Bluetooth communication is from $178 \mu \mathrm{A}$ to $50 \mathrm{~mA}$. The consumption power value of Bluetooth LE communications is from $124 \mu \mathrm{A}$ to $266 \mu \mathrm{A}$. It is definite that the operating power of $\mathrm{Wi}-\mathrm{Fi}$ is a thousand times higher than Bluetooth LE communications. From a software point of view, the difference of power consumption is also obvious. Therefore, in this system, GCM Push and BLE communication are preferred for sending an emergency oneway notification to conserve power, and Wi-Fi communications is used for ending the specific solution among the server and clients because a large amount of data exchange is required in this process.

\section{acknowlegement}

This research was supported by The Leading Human Resource Training Program of Regional Neo industry through the National Research Foundation of Korea(NRF) funded by the Ministry of Science, ICT and future Planning(NRF-2016H1D5A1909408), and the BK21 Plus Program(216C000070) funded by the Ministry of 
Education(MOE, Korea) and National Research Foundation of Korea(NRF)

\section{References}

[1] Hongbeom Ahn, Pyeongjae Lee, Jinpyo Hong. "An Algorithm of Localization by using the Score of Multiple Power Beacon Signals in Wireless Sensor Networks", Korea Computer Congress 2010, Vol. 37(1)(D), 2010.6, 165-169

[2] William Stallings. "Data \& Computer Communication 10th edition". Pearson Education, Inc, 2014.

[3] In-Yeup Kong, Ho-Jin Kim, "Experiments and its analysis on the Identification of Indoor Location by Visible Light Communication using LED lights" Journal of the Korea Institute of Information and Communication Engineering, vol. 15, no. 5, pp. 1045-1052, 2011.

[4] Deepak Vasisht, Swarun Kumar, Dina Katabi, "Decimeter-Level Localization with a SingleWiFi Access Point", 13th USENIX Symposium on Networked Systems Design and Implementation (NSDI' 16)

[5] OnkarPathak, Pratik Palaskar, Rajesh Palkar, Mayur Tawari. "Wi-Fi Indoor Positioning System Based on RSSI Measurements from Wi-Fi Access Points -A Tri-lateration Approach". International Journal of Scientific \& Engineering Research, Volume 5, Issue 4, April-2014

[6] "WL18xxMOD WiLink ${ }^{\mathrm{TM}} 8$ Single-Band Combo Module - Wi-Fi®, Bluetooth $\AA$, and Bluetooth Low Energy (LE)", http://www.ti.com

[7] Google Cloud Messaging (GCM) Push, https://developers.google.com/cloud-messaging/

[8] “Wireless LAN-Based LBS Services”, Hakyong KIM, Apr, 2006

[9] "Bluetooth Indoor Localization System", Gunter FISCHER, Burkhart DIETRICH, Frank WINKLER

[10] Qian Dong, Waltenegus Dargie "Evaluation of the Reliability of RSSI for Indoor Localization" Aug. 2012 